\title{
COMPROMISO EN EL TRABAJO Y PRÁCTICAS DE RECURSOS HUMANOS DE ALTO RENDIMIENTO EN ORGANIZACIONES DE ACCIÓN SOCIAL. EL CASO DE ASPROMANIS
}

\author{
Rosa Benítez SAÑa \\ Ana Rosa del Águila Obra \\ Universidad de Málaga
}

DOI: $10.1387 /$ lan-harremanak.15407

\section{ABSTRACT}

Los empleados con alto nivel de work engagement (WE) se sienten con altos niveles de energía, entusiasmo y concentración en su trabajo. Las organizaciones pueden potenciar el WE implementando prácticas de recursos humanos de alto rendimiento (prácticas STAR), tales como politicas de selección y formación, evaluación del desempeño, politicas retributivas, participación, comunicación, empowerment, conciliación de vida familiar, estabilidad laboral y relaciones interpersonales, entre otros, relacionadas directamente con la calidad en el empleo. Dada la escasa existencia de estudios sobre WE y las prácticas STAR en entidades del Tercer Sector de la economía, concretamente en el ámbito de la acción social, se ha analizado el caso de la Asociación Malagueña en favor de las Personas con Discapacidad Intelectual (Aspromanis). La hipótesis de partida del trabajo consiste en identificar la relación entre el WE de los trabajadores de una organización de acción social, Aspromanis, y la implementación de prácticas STAR. De la investigación realizada se puede concluir que en la organización se observan altos niveles en promedio global de WE, y que las prácticas STAR están ejerciendo una 
influencia positiva en el mismo, en sus tres dimensiones, vigor, dedicación y absorción. Como contribución, se aporta evidencia de que una mayor calidad en el empleo se traduce en altos niveles de WE.

Palabras clave: Compromiso en el trabajo, participación, empowerment, estabilidad laboral y acción social.

Employees with high level of Work Engagement (WE) experience high levels of energy, enthusiasm, and concentration in their jobs. Organizations can potentiate WE through the adoption of new human resources (HR) practices, known as STAR practices, such as selection and training programs, compensation policies, participation, communication, empowerment, job stability and interpersonal relationships, among others, directly related with the job quality. Given the limited existence of studies about WE and HR practices in the Third Sector, particularly in the field of social action, this research analyzes the case of the Malaga Association for Persons with Intellectual Disability (Aspromanis). The main hypothesis was to identify the relationship between the WE, in Aspromanis, and the implementation of STAR practices. This work identifies that there is a positive relation between high levels of WE and the implementation of STAR practices. As contribution, the evidence that a higher quality employment results into higher levels of WE.

Key Words: Work Engagement, Human Resources Practices, Empowerment, Job stability, Social action 


\section{Concepto y dimensiones del compromiso en el trabajo}

En relación al concepto de work engagement (WE) destaca en primer lugar que no existe una traducción del término propiamente dicha. Salanova y Schaufeli (2009), señalan que se trata de un conjunto de conceptos unidos que finalmente lo definen, por lo tanto para hablar de WE hay que referirse a una serie de notas definitorias, relacionadas con el trabajo que se realiza, que nos aproximan al concepto, como son: compromiso, vinculación, satisfacción personal, pasión, entusiasmo, esfuerzo y energía.

Una visión parecida es la que proporcionan Rodríguez-Muñoz y Bakker (2013), quienes señalan la existencia de conceptos similares al WE en castellano, como pueden ser «compromiso o implicación laboral»o «vinculación con el trabajo». También para Salanova y Schaufeli (2004) el término engagement no significa exactamente lo mismo que otros conceptos con los que está relacionado y que sin embargo sí tienen sus correspondientes homónimos en inglés como son: implicación en el trabajo (work involvement), compromiso organizacional (organizational commitment), dedicación al trabajo (work dedication), enganche (work attachment), o adicción al trabajo (workaholism).

Para la mayoría de los investigadores ninguna de las traducciones ofrece una imagen fiel de lo que supone el término, motivo por el cual en el ánimo de no desvirtuar sus múltiples connotaciones, casi todos han preferido mantener el uso del término en su idioma original.

La definición más extendida y ampliamente utilizada, según se desprende de la revisión bibliográfica, es la aportada por Schaufeli y otros (2002:74) que lo definen como «un estado mental positivo relacionado por el trabajo y caracterizado por vigor, dedicación y absorción. Más que un estado específico y momentáneo, el engagement se refiere a un estado afectivo-cognitivo que no está focalizado en un objeto, evento o situación particular».

Por su parte, Salanova y otros (2005) consideran el engagement como «un constructo claramente motivacional ya que posee componentes de activación, energía, esfuerzo y persistencia y está dirigido a la consecución de objetivos». Las personas que experimentan engagement sienten estar fuertemente vinculados con sus tareas y tienen la percepción de que son capaces de afrontarlas con 
total eficacia, respondiendo así a las demandas del puesto de trabajo. Además de ello, los trabajadores engaged disfrutan ejecutando sus tareas y experimentan emociones placenteras de plenitud y autorrealización relacionadas con las mismas.

En esta misma línea, se encuentran otras definiciones relativamente más recientes, las cuales reflejan el WE como una experiencia vital positiva de los trabajadores en su ámbito laboral, por ejemplo para Bakker y otros (2011) los trabajadores con engagement se encuentran llenos de energía y con deseos de destinar esfuerzo y tiempo a sus tareas diarias (vigor), sienten que éstas están dotadas de significancia y valor y además consideran que su trabajo supone un desafío, interesante y apasionante (dedicación) sienten que el tiempo "pasa volando" mientras trabajan y les cuesta abandonar la tarea (absorción).

Durán y Manteca (2013) nos aproximan recientemente a una traducción al castellano del término, al que añaden además la «vinculación psicológica con el trabajo» 0 «ilusión por el trabajo».

Una vez tenidas en cuenta todas estas definiciones, se puede afirmar que el WE es un constructo que se desarrolla en tres dimensiones: vigor, dedicación y absorción. Es un estado mental positivo y persistente en que se encuentra el trabajador mientras realiza su trabajo. El trabajador, durante su jornada laboral se encuentra feliz, lleno de energía y empuje para llevar a cabo sus tareas (vigor). Posee además un fuerte vínculo emocional positivo hacia su trabajo del que se siente orgulloso porque le entusiasma, lo encuentra lleno de significado y supone además un desafío y fuente de inspiración para él (dedicación). Finalmente, el trabajador tiene la sensación de que durante la realización de su trabajo el tiempo "pasa volando" y este le resulta tan gratificante que incluso le cuesta abandonar la tarea en la que se encuentra altamente concentrado (absorción). En definitiva, el trabajador siente verdadera pasión por su trabajo por lo que no le cuesta trabajo invertir esfuerzo extra en realizarlo.

En resumen, el WE, o compromiso en el trabajo, consiste en un estado mental positivo en el que se encuentra el empleado al realizar su trabajo, sintiéndose con altos niveles de energía, entusiasmo y concentración. Por otra parte se trata de un concepto tridimensional (vigor, dedicación y absorción), que presenta diversos factores potenciadores del mismo.

\section{Prácticas organizacionales de gestión de personas}

Las organizaciones, para hacer frente a un entorno económico adverso como el actual pueden potenciar el WE implementando prácticas de gestión de personas basadas en Sistemas de Trabajo de Alto Rendimiento (prácticas STAR) (po- 
líticas de selección, formación, evaluación del desempeño, políticas retributivas, participación, comunicación, empowerment, conciliación de vida familiar, estabilidad laboral y relaciones interpersonales), en otros, términos, a través de la calidad del empleo.

En suma, las prácticas STAR, conocidas en inglés como High Performance Work Systems (HPWS), consisten en llevar a cabo acciones en materia de gestión de personas que fomenten la participación e implicación de los empleados y que favorezcan la mejora de la calidad de vida en el trabajo y el bienestar del trabajador. En este sentido, Chuang y Liao (2010) concluyen que, además de lo concerniente a los trabajadores, este tipo de prácticas mejoran los resultados de una unidad de negocio y además asocian su influencia directa con la efectividad en el contexto del servicio al cliente.

Por otra parte y complementariamente a lo anterior, diversos estudios sobre las organizaciones saludables, dentro del marco de la Psicología de la Salud Ocupacional Positiva, ponen su foco de atención en el impacto positivo de las Prácticas Organizacionales Saludables, desde la Dirección de Recursos Humanos, como potenciadoras del WE de los trabajadores. Estas prácticas cobran vital importancia para las organizaciones por ser responsables del incremento de los niveles de confianza organizacional (Acosta et al., 2011) y del WE (Torrente et al., 2012).

Partiendo desde el enfoque de la Psicología Positiva, refiriéndose a las prácticas de Recursos Humanos en las organizaciones, Durán y Manteca (2013), afirman que mediante la aplicación y desarrollo de programas cuyo enfoque se basa en la Psicología de la salud Ocupacional Positiva, las organizaciones consiguen reducir la incidencia de psicopatologías asociadas al ámbito laboral y mejorar la calidad de vida de los trabajadores que las componen.

Este punto de vista se relaciona con el de Luthans (2002) quien hace hincapié en la necesidad del «estudio y aplicación de recursos y competencias humanas que pueden ser medidas, desarrolladas y gestionadas con el objetivo de mejorar el desempeño en las organizaciones». Estos enfoques citados, coinciden además con lo que plantean Salanova y Schaufeli (2004), quienes afirman que la mejora del desempeño pasa por la mejora de la calidad de vida laboral, para ello las organizaciones que apliquen a la hora de dirigir los recursos humanos prácticas que se basen en potenciar constructos positivos como el WE tendrán mayor éxito en sus propósitos.

Sobre todo, si se parte de la base de que las personas que integran las organizaciones son su factor clave, mantener su salud y bienestar y favorecer su desarrollo personal y profesional supondrá una ventaja competitiva para las mismas. Así lo señalan Salanova y Schaufeli (2004), para ellos es mucho mejor invertir dinero y esfuerzo en optimizar la efectividad, el desarrollo de los em- 
pleados que tener que afrontar problemas evitables y económicamente más costosos a la larga (absentismo, disminución de la productividad, etc). En la medida en que se favorezca la mejora del ambiente de trabajo se favorecerá también el WE, y con ello se obtendrán «beneficios adicionales que son positivos para la organización» (Salanova y Schaufeli, 2004:121).

Dentro del marco teórico de las Organizaciones Saludables y Resilientes (Healthy and Resilient Organizations, HERO, por sus siglas en inglés), se identifica que las organizaciones que pueden responder a este modelo son aquellas que de manera proactiva hacen esfuerzos sistemáticos y planificados por mejorar la salud de sus empleados y de la organización a través de prácticas organizacionales saludables (Acosta et al., 2011). Según estos autores, las prácticas se realizan en tres niveles: nivel organizacional (conciliación trabajo-familia, prácticas organizacionales para la mejora de la salud), nivel de la tarea (rediseńo de tareas para mejorar la autonomía, feedback) y nivel del ambiente social (liderazgo). Además para ellos las prácticas organizacionales constituyen un elemento clave del modelo junto con los recursos laborales.

En opinión de Acosta y otros (2011), para el desarrollo de una organización saludable se puede acudir a prácticas tales como formación de habilidades de liderazgo, feedback, comunicación, uso de redes intranet, prevención y gestión del estrés, selección cuidadosa del personal, conciliación de vida laboral y familiar, flexibilidad laboral, evaluación del desempeño, etc.

Estos autores categorizan las prácticas organizacionales saludables, en las siguientes áreas (Acosta et al., 2011):

1. Recursos Humanos: desarrollo de habilidades, desarrollo de carrera, prevención y gestión del mobbing, equidad, selección de personal, retribuciones, evaluación del desempeño.

2. Salud y seguridad en el trabajo: prácticas por ley, iniciativa de la empresa y estabilidad laboral.

3. Gestión del impacto ambiental: reciclaje y consumo energético.

4. Inserción de personas en riesgo de exclusión social: contratación de personas en riesgo de exclusión social.

5. Comunidades locales: intervención de las empresas en su comunidad.

6. Igualdad de oportunidades: conciliación trabajo-familia y flexibilidad laboral.

7. Comunicación e información organizacional: ascendente, descendente y horizontal.

8. Códigos de conducta: manuales y protocolos, normas y sanciones.

9. Relaciones interpersonales: fomento de relaciones interpersonales en el clima laboral.

10. Confianza organizacional: vertical, horizontal y clientes. 
De forma similar, Tang y Tang (2012) y Stirpe y Revilla (2013), clasifican las prácticas de recursos humanos basadas en sistemas de trabajo de alto rendimiento en seis áreas de acción fundamentales: políticas de selección, formación, evaluación del desempeño, políticas retributivas, participación, y empowerment.

Por su parte Reissner y Pagan (2012), incorporan otras prácticas como el storytelling organizacional, y las asocian con niveles altos de WE, destacando su papel clave para la comunicación de la cultura y valores de la organización y su influencia positiva en la implicación y sentido de pertenencia de los empleados.

Como conclusión, respecto a la prácticas organizacionales y su influencia en el WE, se puede afirmar, según se desprende de lo expuesto hasta ahora, que con la aplicación de prácticas organizacionales basadas en sistemas STAR, se favorece el WE y a su vez el bienestar y mejora del ambiente de trabajo, obteniéndose ventajas, tanto a nivel individual y organizacional, que redundan en un mayor rendimiento laboral y resultados organizacionales.

\section{El caso de Aspromanis}

Dada la escasa existencia de estudios sobre WE y prácticas STAR en entidades del Tercer Sector de la economía, concretamente en el ámbito de la acción social, se ha analizado el caso de la Asociación Malagueña en favor de las Personas con Discapacidad Intelectual (Aspromanis).

La hipótesis de partida del trabajo consiste en identificar la relación entre el WE de los trabajadores de una organización de acción social, Aspromanis, y la implementación de prácticas STAR.

Los datos utilizados para la realización de la investigación proceden por un lado de dos entrevistas abiertas no estructuradas, con el gerente y la persona responsable del área de relaciones laborales y retribuciones de la entidad, tomándose como guía una combinación, ad-hoc, de las prácticas STAR (Stirpe y Revilla,(2013), y de la categorización de prácticas organizacionales saludables (Acosta et al., 2011). A partir de la información obtenida se realizó un estudio analítico-descriptivo con el objeto de identificar las prácticas STAR puestas en marcha por la dirección de la entidad.

Por otra parte y para la medición del WE se procedió a encuestar a 42 profesionales de Aspromanis (33,66 por ciento de un total de 125 trabajadores de los que se compone su plantilla total). La recopilación de datos tuvo lugar en el transcurso de una reunión de profesionales de la asociación, celebrada en el mes de Diciembre de 2013, en la cual se obtuvieron 35 encuestas. Posteriormente, dado el interés en participar manifestado por la dirección de otro centro de trabajo de la Asociación, en enero de 2014 se distribuyó el cuestionario a un grupo 
de 7 trabajadores más que forman parte de dicho centro, pasando a integrarse en la muestra, resultando finalmente un total de 42 trabajadores. Para medir los niveles de WE existentes en la organización, se utilizó como herramienta de medición la encuesta UWES-17 de la Universidad de Utrecht.

\subsection{Contexto}

Aspromanis es una entidad sin ánimo de lucro, declarada de utilidad pública y perteneciente al Tercer Sector de la economía, cuya actividad se encamina a contribuir a la mejora de la calidad de vida de las personas con discapacidad intelectual y sus familias, prestando para ello servicios de apoyo en todos los ámbitos de su vida. Aspromanis cuenta con plazas concertadas con la Junta de Andalucía en centros de día y residenciales establecidos en toda la provincia de Málaga y su participación en la sociedad malagueña se remonta a cincuenta años atrás.

Aspromanis considera el capital humano de la organización un factor estratégico y generador de ventajas competitivas de la entidad desde sus inicios. Se ha evidenciado este aspecto en mayor medida en los últimos años con la puesta en marcha en 2010 de un Plan de Calidad para la implantación de un Sistema de Gestión de la Calidad basado en el modelo EFQM ${ }^{1}$ y el Sistema de Calidad FEAPS 2 .

En línea con ambos sistemas, promovido por el Departamento de Gestión de Personas de la entidad y en colaboración con un equipo ad-hoc para la ocasión, integrado por trabajadores y sus representantes legales, así como personal directivo, se realizó en 2012 un estudio para la elaboración de su primer Plan de Personas basado en el método estratégico de gestión de personas, denominado LIDER, que a su vez forma parte del Plan Estratégico de Aspromanis 2012$2015^{3}$. En este plan se inserta entre sus objetivos clave conseguir el desarrollo profesional y la mejora en la cualificación de sus trabajadores, a través de planes formativos adaptados a cada necesidad, se aspira con ello a mantener la mejora continua en la calidad del servicio que se presta, orientado a sus principales clientes que son las personas con discapacidad intelectual y sus familias.

\subsection{Prácticas STAR en Aspromanis}

Con el objeto de conocer las prácticas STAR puestas en marcha por Aspromanis, se realizaron dos entrevistas abiertas no estructuradas al gerente y la responsable del área de relaciones laborales y retribuciones de la organización,

\footnotetext{
1 EFQM: European Foundation of Quality Management.

2 Calidad FEAPS: Sistema de Gestión de la Calidad de la Confederación Española de Organizaciones en favor de las Personas con Discapacidad Intelectual.

3 Plan Estratégico de Aspromanis 2012-2015. Disponible en http://www.aspromanis.es/
} 
para ello se tomó como guía una combinación ad-hoc basada en la encuesta de Prácticas STAR diseñada por Stirpe y Revilla (2013), la clasificación de Tang y Tang (2012) de las prácticas de Recursos Humanos de alto rendimiento y las prácticas en organizacionales saludables, según el modelo HERO propuesto por Acosta y otros (2011). Resultando de estas entrevistas una identificación categorizada de prácticas STAR habitualmente utilizadas por la entidad, según muestra el cuadro 1.

\section{Cuadro 1}

Prácticas STAR en Aspromanis

\begin{tabular}{|c|c|}
\hline $\begin{array}{l}\text { Área de acción } \\
\text { de las prácticas } \\
\text { STAR }\end{array}$ & Acciones utilizadas \\
\hline $\begin{array}{l}\text { Políticas de } \\
\text { selección }\end{array}$ & $\begin{array}{l}\text { - Procesos de selección rigurosos cuidando al máximo la adecuación de } \\
\text { la titulación al puesto a ocupar. } \\
\text { — Procesos de selección mediante equipo multidisciplinar (assessment } \\
\text { center) en los puestos clave de la organización. } \\
\text { _ Ofertas de participación en las actividades selectivas para la promo- } \\
\text { ción interna de los empleados. } \\
\text { — Procesos de acogida de los nuevos empleados, mediante tutorización } \\
\text { por otro empleado o equipo de trabajo. }\end{array}$ \\
\hline Formación & $\begin{array}{l}\text { - Planes de formación destinados a la plantilla al completo, tanto en } \\
\text { formación específica como polivalente. } \\
\text { — Planes de cualificación profesional de acuerdo el Plan Acredita } 2014 \\
\text { y con la Clasificación Nacional de Ocupaciones. } \\
\text { _ Grupos de trabajo para la gestión del conocimiento interdepartamen- } \\
\text { tal e interindividual. }\end{array}$ \\
\hline $\begin{array}{l}\text { Evaluación del } \\
\text { Desempeño }\end{array}$ & $\begin{array}{l}\text { - Sistema de evaluación del desempeño a } 90^{\circ}, 180^{\circ} \text { y } 360^{\circ} \text { depen- } \\
\text { diendo del puesto de trabajo que desempeñan. } \\
\text { - Procedimientos de consenso en evaluaciones } 360^{\circ} \text {, conformidad del } \\
\text { trabajador evaluado y alegaciones. }\end{array}$ \\
\hline $\begin{array}{l}\text { Políticas } \\
\text { retributivas }\end{array}$ & $\begin{array}{l}\text { - Aplicación exhaustiva del convenio colectivo en las retribuciones sala- } \\
\text { riales. } \\
\text { — Acuerdo inter-partes para el abono de los festivos trabajados en ma- } \\
\text { yor cuantía que la establecida en el convenio colectivo. }\end{array}$ \\
\hline Participación & $\begin{array}{l}\text { - Creación de círculos de calidad con participación activa en la rees- } \\
\text { tructuración organizativa con motivo de la implantación de los siste- } \\
\text { mas Gestión de la Calidad y la mejora continua. } \\
\text { - Participación en reuniones y debates para la evaluación del servicio y } \\
\text { aportación de nuevas ideas. }\end{array}$ \\
\hline
\end{tabular}




\begin{tabular}{|c|c|}
\hline Comunicación & $\begin{array}{l}\text { - Difusión del Plan Estratégico de Aspromanis. } \\
\text { — Difusión Cuentas Anuales publicadas en web. } \\
\text { - Difusión de INFO Aspromanis en toda la plantilla. } \\
\text { — Página web corporativa con difusión de la misión, visión y valores de } \\
\text { la entidad. } \\
\text { — Difusión del Código Ético de Aspromanis, presente en todos los cen- } \\
\text { tros de trabajo y página web. } \\
\text { - Difusión información relevante en Tablones de Anuncios. } \\
\text { - Buzones de sugerencias constructivas para uso general de la Asocia- } \\
\text { ción y Banco de Ideas (informal). }\end{array}$ \\
\hline \multicolumn{2}{|r|}{$\begin{array}{c}\text { Cuadro } 1 \\
\text { (continuación) }\end{array}$} \\
\hline $\begin{array}{l}\text { Área de acción } \\
\text { de las prácticas } \\
\text { STAR }\end{array}$ & Acciones utilizadas \\
\hline Empowerment & $\begin{array}{l}\text { - Autonomía en la toma de decisiones de los empleados. } \\
\text { — Equipos autónomos para resolución de problemas y toma de decisio- } \\
\text { nes. Equipos de Trabajo de Alto Rendimiento. }\end{array}$ \\
\hline $\begin{array}{l}\text { Conciliación } \\
\text { de vida familiar }\end{array}$ & $\begin{array}{l}\text { - Especial protección de los riesgos laborales durante el embarazo: } \\
\text { adaptación del puesto y suspensión del contrato en caso de no ser via- } \\
\text { ble la adaptación. } \\
\text { — Reducciones de jornada por cuidado de hijos y familiares. } \\
\text { — Excedencias por cuidado de hijos y familiares. } \\
\text { — Medidas de flexibilización horaria por solicitud de los trabajadores. }\end{array}$ \\
\hline $\begin{array}{l}\text { Estabilidad } \\
\text { laboral }\end{array}$ & $\begin{array}{l}\text { - Alto índice de empleo estable, contratación indefinida del } 85 \text { por } \\
\text { ciento de la plantilla. }\end{array}$ \\
\hline $\begin{array}{l}\text { Relaciones } \\
\text { interpersonales }\end{array}$ & $\begin{array}{l}\text { - Fomento del buen clima laboral y relaciones interpersonales mediante } \\
\text { reuniones de cohesión de grupos, debate y dinámicas de grupo que } \\
\text { incluyen métodos de narración de experiencias. }\end{array}$ \\
\hline
\end{tabular}

Fuente: Elaboración propia.

Cabe destacar por su especial relevancia, que la entidad, a la fecha de realización de este trabajo de investigación, mayo 2014, estaba inmersa en la implantación de un sistema de Evaluación del Desempeño. Su puesta en marcha, tiene su origen en la aplicación del acuerdo consensuado por la patronal y los sindicatos del sector y publicado en el XIV Convenio Colectivo sectorial-nacional $^{4}$. Lo cual es un dato importante, dado que este tipo de consenso en ne-

${ }^{4}$ XIV Convenio Colectivo General de Centros de Atención a Personas con Discapacidad. Disponible en: http://www.boe.es/boe/dias/2012/10/09/pdfs/BOE-A-2012-12618.pdf 
gociación colectiva no suele ser muy habitual, y mucho menos a nivel tan amplio. Por regla general, son las empresas, por iniciativa propia, las que suelen decidir la aplicación de estos sistemas. En este caso, el convenio colectivo vincula el sistema de evaluación del desempeńo a las subidas salariales a aplicar a los trabajadores. No obstante, Aspromanis ha querido ir un paso más allá y, dejando esta vinculación a la subida salarial en un plano secundario, ha decidido enfocar principalmente este procedimiento de evaluación del desempeño como una fuente de identificación de necesidades formativas encaminadas hacia el desarrollo profesional y personal de sus profesionales, basado en la mejora continua, estableciendo así un vínculo directo con los sistemas de gestión de la calidad.

\subsection{Medición del compromiso en el trabajo en Aspromanis}

Para efectuar la medición del WE se adoptó como herramienta la Utrecht Work Engagement Scale (UWES-17), concretamente la adaptación española realizada en el Manual UWES, adaptándose además el cuestionario 5 para Aspromanis. En esta investigación ha participado una muestra compuesta por 42 profesionales que trabajan en la Asociación Malagueña en favor de las Personas con Discapacidad Intelectual-Aspromanis, que suponen el 33,66 por ciento de un total de 125 trabajadores de los que se compone su plantilla total. La estructura de la muestra ha sido heterogénea y representativa en su composición, por este motivo se encuentran incluidos en ella trabajadores de distintas ocupaciones, nivel de estudios, edad y antigüedad en la organización, así como personal de distintos centros y áreas de trabajo del conjunto de la asociación. El nivel de adecuación de la muestra ha sido establecido en un 95 por ciento de validez con un índice de error del 5 por ciento. En el cuadro 2 se puede apreciar la estructura de la muestra así como los perfiles socio-demográficos de distribución por variables categóricas utilizadas para el estudio.

El análisis de datos se realizó mediante el paquete estadístico SPSS versión 22.0 y la herramienta de cálculo de Microsoft Excel, llevándose a cabo análisis descriptivos (media y desviación típica) y de consistencia interna (Alpha de Cronbach).

5 Ver Anexo I. Encuesta UWES 17 adaptada para Aspromanis. 


\section{Cuadro 2}

Variables socio-demográficas de la muestra de trabajadores Aspromanis

\begin{tabular}{|c|c|c|c|c|c|c|c|}
\hline \multicolumn{4}{|c|}{ Variables sociodemográficas } & \multicolumn{4}{|c|}{ Perfiles por género } \\
\hline Variable & Categoría & $N=$ & $\begin{array}{c}\% \text { s/ } \\
\text { muestra }\end{array}$ & Hombres & $\begin{array}{c}\% \text { s/ } \\
\text { muestra }\end{array}$ & Mujeres & $\begin{array}{c}\% \mathrm{~s} / \\
\text { muestra }\end{array}$ \\
\hline \multirow{4}{*}{$\begin{array}{l}\text { Ámbito de } \\
\text { ocupación }\end{array}$} & Atención directa & 26 & 61,90 & 10 & 38,46 & 16 & 61,54 \\
\hline & Técnico específico & 6 & 14,29 & 1 & 16,67 & 5 & 83,33 \\
\hline & Administrativo & 7 & 16,67 & 1 & 14,29 & 6 & 85,71 \\
\hline & Servicio doméstico & 3 & 7,14 & 0 & 0,00 & 3 & 100,00 \\
\hline \multirow{4}{*}{$\begin{array}{l}\text { Nivel de } \\
\text { estudios }\end{array}$} & Primarios & 8 & 19,05 & 3 & 37,50 & 5 & 62,50 \\
\hline & Secundarios & 17 & 40,48 & 4 & 23,53 & 13 & 76,47 \\
\hline & Diplomatura & 13 & 30,95 & 5 & 38,46 & 8 & 61,54 \\
\hline & Licenciatura & 4 & 9,52 & 0 & 0,00 & 4 & 100,00 \\
\hline $\begin{array}{l}\text { Media de } \\
\text { edad }\end{array}$ & \multicolumn{3}{|c|}{41,40} & 47 & \multicolumn{3}{|c|}{40} \\
\hline Antigüedad & \multicolumn{3}{|c|}{12,00} & 15,75 & \multicolumn{3}{|c|}{10,58} \\
\hline \multicolumn{2}{|c|}{ Total muestra } & 42,00 & 100,00 & 12,00 & 28,57 & 30,00 & 71,43 \\
\hline
\end{tabular}

Fuente: Elaboración propia.

En cuanto a la medición por promedios realizada en la entidad Aspromanis, se presentan en el Cuadro 3 los resultados del nivel promedio de WE existente, tanto de manera global o unidimensional (WE global), como por cada una de sus dimensiones (vigor, dedicación y absorción). Estos niveles se comparan en dicha tabla con el baremo normativo de UWES-17 adaptado para España de Schaufeli y Bakker (2003). Estos resultados reflejan niveles altos de WE global, así como también niveles altos en cada una de las dimensiones medidas en las subescalas vigor, dedicación y absorción. 
Cuadro 3

Resultados del WE en Aspromanis comparados con el baremo español

\begin{tabular}{lcccc}
\hline \multicolumn{5}{c}{ UWES-17 N= 1275 trabajadores españoles } \\
\hline \multicolumn{1}{c}{$\begin{array}{c}\text { Puntuaciones } \\
\text { Baremo }\end{array}$} & Vigor & Dedicación & Absorción & $\begin{array}{c}\text { Engagement } \\
\text { Global }\end{array}$ \\
\hline Rango & $0.00-6.00$ & $0.00-6.00$ & $0.00-6.00$ & $0.00-6.00$ \\
Muy bajas & $\leq 2.17$ & $\leq 1.60$ & $\leq 1.60$ & $\leq 1.93$ \\
Bajas & $2.18-3.20$ & $1.61-3.00$ & $1.61-2.75$ & $1.94-3.06$ \\
Moderadas & $3.21-4.80$ & $3.01-4.90$ & $2.76-4.40$ & $3.07-4.66$ \\
Altas* & $4.81-5.60$ & $4.91-5.79$ & $4.41-5.35$ & $4.67-5.53$ \\
Muy altas & $\leq 5.61$ & $\leq 5.80$ & $\leq 5.36$ & $\leq 5.54$ \\
\hline Puntuación media Baremo español & 3.99 & 3.81 & 3.56 & 3.82 \\
\hline \multicolumn{5}{c}{ Resultados UWES-Aspromanis N $=42$ trabajadores } \\
\hline Puntuación media ASPROMANIS* & 5.35 & 5.51 & 4.91 & 5.27 \\
\hline
\end{tabular}

* Puntuación media de Aspromanis comprendida en el rango de puntuaciones altas según baremo.

Fuente: Schaufeli y Bakker (2003) y elaboración propia.

En lo relativo a los resultados de WE teniendo en cuenta el género de los empleados, ver figura 1, en ambos casos se mantienen niveles altos de WE, no obstante las mujeres presentan un nivel de 5,35 que es ligeramente más alto que los hombres con una puntuación media de 4,95.

Figura 1

Nivel de work engagement global según género

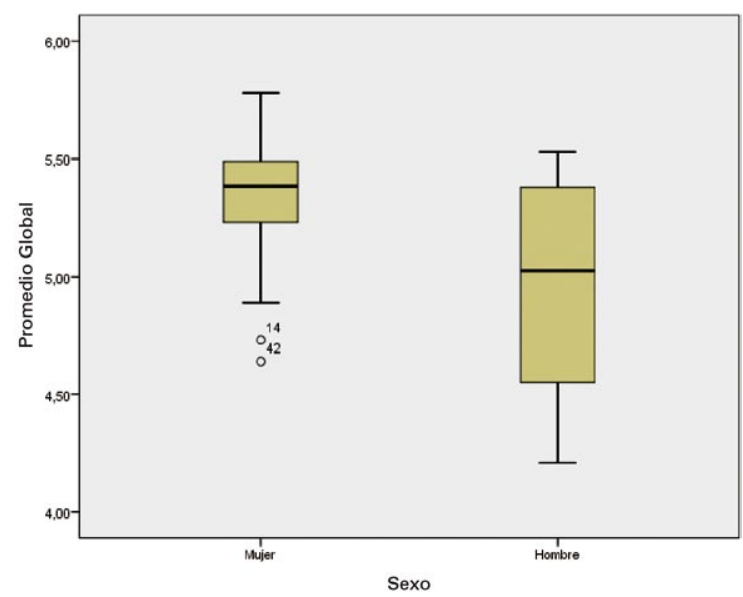

Fuente: Elaboración propia. 
La puntuación en promedio de WE por ámbito profesional, ver figura 2, se sitúa en niveles más bajos en el caso de los empleados de servicio doméstico.

\section{Figura 2}

Resultados de work engagement según ámbito profesional

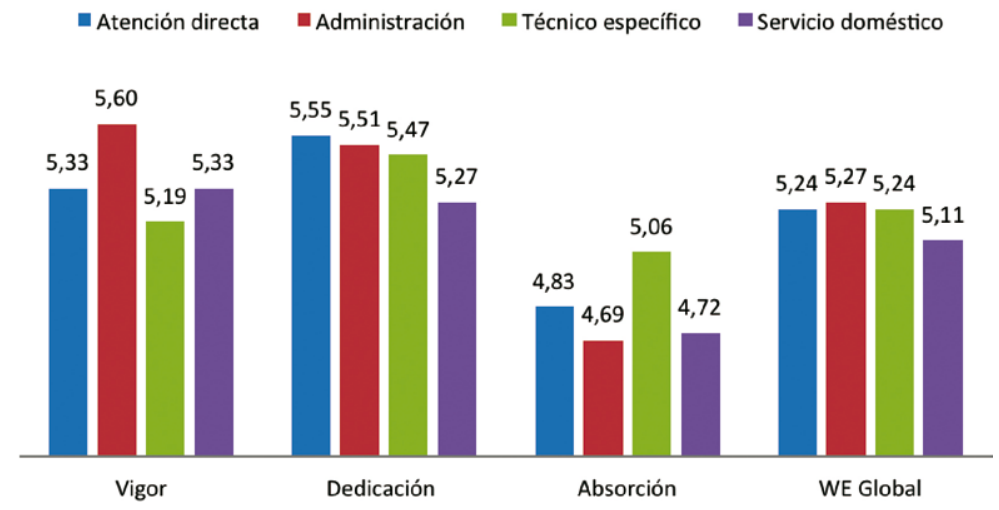

Fuente: Elaboración propia.

Asimismo, a la hora de valorar los resultados en función de la edad de los trabajadores encuestados, ver figura 3, se observa que el promedio de WE se mantiene en nivel alto sin diferencias apreciables entre rangos de edad, tan solo se muestra un leve ascenso, pero aun manteniéndose en niveles altos, en el rango de edad comprendida entre 41 y 50 años de edad.

Figura 3

Resultados de work engagement por rango de edad

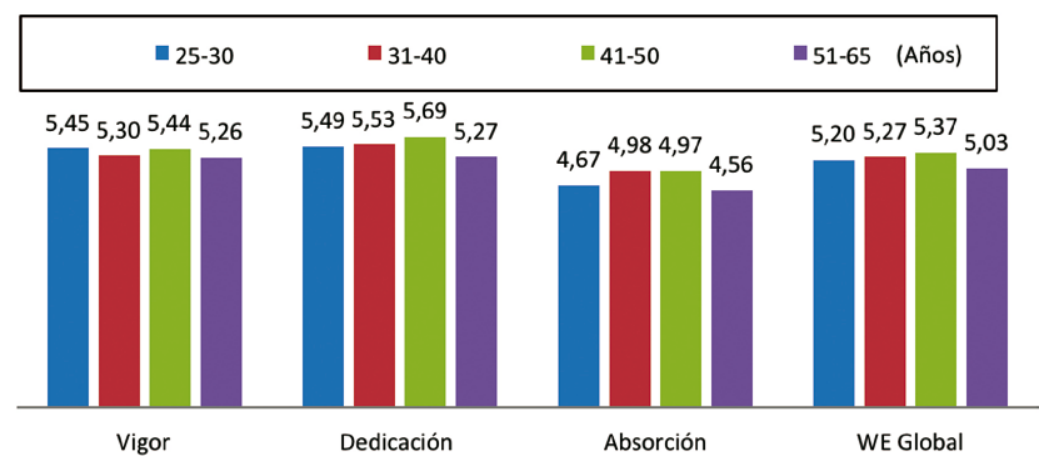

Fuente: Elaboración propia. 
Los niveles de WE tomados según la antigüedad en la organización de los trabajadores participantes, ver figura 4, se mantienen en niveles altos, tanto en puntuación global como en sus tres dimensiones. Cabe destacar el rango de empleados con permanencia entre 11 y 20 ańos de antigüedad en la entidad, cuya puntuación es más baja en promedio global, así como en las tres dimensiones, aun así este grupo sigue manteniéndose en niveles altos en vigor y absorción. Sin embargo su puntuación es la más baja en absorción y se sitúa en niveles moderados según la comparación con el baremo normativo en esta dimensión.

Sin embargo los trabajadores comprendidos entre los 6 y 10 años de antigüedad en la organización son los que obtienen resultados ligeramente más altos en las tres dimensiones y en WE global, permaneciendo en puntuaciones altas de WE.

Figura 4

Resultados de work engagement según la antigüedad en la organización

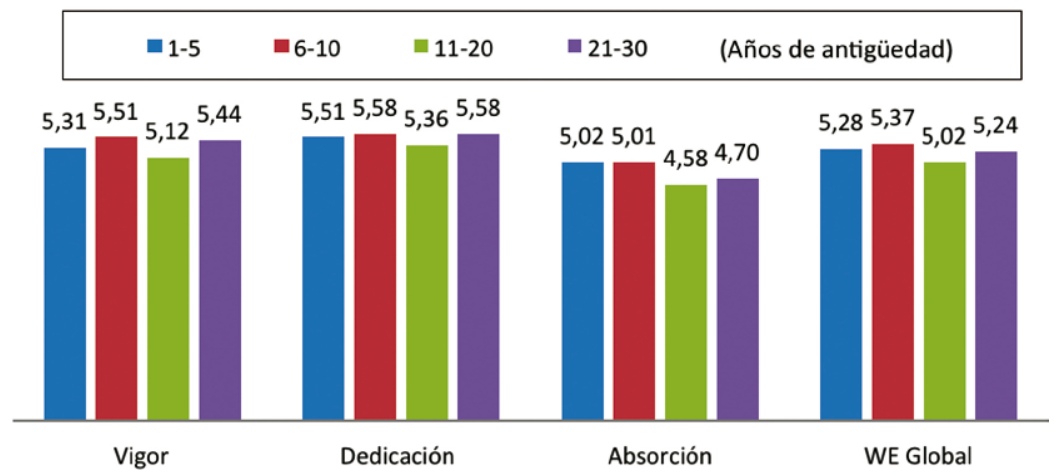

Fuente: Elaboración propia.

\subsection{Análisis de resultados y discusión}

En cuanto a los resultados de la medición del WE, al ser éstos comparados con el baremo normativo español de UWES-17, se obtiene que en nivel global así como en todas sus dimensiones, las puntuaciones se sitúan en niveles altos. Los profesionales encuestados en la muestra de estudio de dicha entidad presentan puntuaciones situadas en un nivel alto en vigor, dedicación y absorción. Estos niveles vienen a coincidir con los resultados del estudio realizado en el ámbito de la acción social por Durán y Manteca (2013), puesto que tanto en términos globales, y en las dimensiones vigor y absorción las puntuaciones se situaron en niveles altos. 
Sin embargo, comparando los resultados de la muestra de Aspromanis, los resultados difieren de los de este estudio, en cuanto a la variable dedicación, cuyo promedio se sitúa en niveles moderados en la muestra de Durán y Manteca (2013) y en niveles altos en la muestra de Aspromanis. Esto podría explicarse porque sus estudios se han centrado en una población muy diversa y de distintas entidades dedicadas al campo de la acción social así como en la Administración, existiendo en esta última mayor estabilidad laboral. No ocurre así, según indican, en las Asociaciones, donde está más presente, según ellos, la inestabilidad laboral y los contratos eventuales y por obra o servicio para realización de proyectos. Este factor influye a la hora de una mayor dedicación, puesto que a veces las contrataciones se realizan para escasos periodos de tiempo, lo cual para la persona contratada puede suponer una desmotivación, ya que no tiene iguales expectativas de futuro que si tuviese mayor estabilidad. En el mencionado estudio de Durán y Manteca (2013) la media de antigüedad de los trabajadores encuestados fue de seis años.

Sin embargo, en el caso de Aspromanis el factor de la estabilidad laboral destaca por lo contrario y se encuentra un resultado distinto que llama la atención especialmente, al poner en relación la dimensión dedicación, que resulta en niveles altos, con el factor de estabilidad laboral que viene determinada por la antigüedad media en el puesto. Como se ha indicado en el apartado relativo a las prácticas organizacionales, Aspromanis desarrolla políticas de empleo estable. Además, según los datos de la muestra, la media de antigüedad es de 12 años de permanencia en el puesto, aunque para ser más precisos, estos resultados sería necesario compararlos con la media de antigüedad de la plantilla total. Aún así, cruzando ambos datos, se puede concluir que la alta estabilidad laboral influye en las puntuaciones altas de dedicación obtenidas a diferencia de otras Asociaciones del sector cuyos índices de dualidad laboral son más altos.

En las asociaciones, según Durán y Manteca (2013) la estabilidad es escasa debido a las políticas de rotación y dualidad en la contratación derivadas de los sistemas de trabajo para proyectos concretos, esto hace que la dimensión dedicación se dé en niveles moderados, sin embargo en Aspromanis los niveles son altos en esta dimensión, lo que permite concluir, como se ha indicado, que esto es debido al alto índice de contratación indefinida que posee la entidad. Esta diferencia se muestra como claro factor diferenciador que podría también ser estudiado en comparación con otros estudios en este sector.

En cuanto a otro de los objetivos de este estudio de caso, el relativo al análisis de las prácticas de gestión de personas implantadas en la Asociación, se puede concluir que éstas parecen estar ejerciendo una influencia positiva en los altos niveles en promedio global de WE, así como en sus tres dimensiones, vigor, dedicación y absorción. Aunque a este respecto, hay que establecer una reserva de- 
bido a que estas prácticas han sido determinadas mediante entrevistas a la $\mathrm{Di}$ rección de la entidad. No obstante, trabajos previos como los de Stirpe y Revilla (2013), Tang y Tang (2012) y Acosta y otros (2011), indican como las prácticas de gestión de personas basadas en STAR y HERO han sido altamente efectivas en las organizaciones que las aplican, generando altos niveles de engagement en sus trabajadores.

\section{Conclusiones}

Esta investigación ha puesto de manifiesto varios aspectos novedosos en relación al WE, por un lado los estudios previos analizados concluyeron que no se apreciaban diferencias significativas en lo que a género se refiere, y sin embargo en esta investigación se presenta una contradicción en este sentido, dado que las mujeres presentan mayores niveles de WE que los hombres. Aun así es un tema a tratar con cautela dada la alta feminización del sector, lo que puede contribuir a estos resultados no esperados.

Otra aportación novedosa del estudio de caso realizado, surge en cuanto a la antigüedad en la empresa, que se relaciona con la estabilidad laboral la cual influye en la dimensión dedicación, puesto que el trabajador está comprometido con su trabajo y se siente orgulloso de realizarlo, en la medida en que siente seguridad en el mismo, al hilo de lo teorizado por Kahn (1990), el trabajador que encuentra significado en el trabajo que hace se siente más comprometido con el mismo y no le importa dedicar tiempo y esfuerzo extraordinario a su realización. Sin embargo, en el estudio de Durán y Manteca (2013) se encontró que en las Asociaciones dedicadas a la acción social los niveles de dedicación eran moderados. Esto también plantea una diferencia destacable en este trabajo empírico ya que en el caso de Aspromanis los niveles de dedicación son altos por lo que esta variable, la estabilidad en el empleo, por tanto la calidad del empleo, se establece claramente como un factor importante a tener en cuenta a la hora de llevar a cabo las políticas de contratación en la organización.

Las limitaciones de este trabajo empírico son varias, en primer lugar habría que profundizar más en los estudios en este sector, ya que son bastante escasos y estos se han realizado en corte transversal, por lo que futuros estudios longitudinales, podrían arrojar mayores datos comparativos sobre el WE, evaluando sus niveles desde un punto de partida y contrastando su evolución en el tiempo. Pudiendo establecerse incluso a nivel metodológico continuo, junto con otras encuestas de satisfacción laboral, burnout, recuperación tras la jornada, etc.

Otra limitación se puede encontrar en el tamaño de la muestra, debido a que la plantilla total de la entidad es un número también reducido, por lo que en el diseño habría que acudir a una muestra mayor e incluso abarcar la totali- 
dad de la plantilla de forma que los datos arrojen resultados más concluyentes. Se podrían también realizar otros estudios para analizar con más profundidad, las correlaciones existentes entre las prácticas de gestión de personas y determinar cuáles son las que más pueden influir en el aumento de los niveles de WE en Aspromanis, para que de esta forma se puedan potenciar si cabe aún más, con los consiguientes beneficios para la organización y las personas.

Por último, en futuras investigaciones podría abordarse estudiar la influencia del WE de los empleados en la calidad del servicio y la satisfacción de los clientes usuarios de los servicios de la entidad, ya que estos factores relacionados entre si no han sido estudiados en este sector concreto dedicado a las personas con discapacidad, y por los datos de este estudio puede intuirse, a nivel inferencial que existe una relación entre ambos aspectos. Además, puede resultar interesante abordarlo de manera más completa ya que en otros estudios realizados sobre clima del servicio y ciudadanía organizacional se han encontrado resultados que apuntan a su relación directa (Tang y Tang, 2012).

En esta coyuntura económica es cuando el WE cobra más sentido si cabe, ya que las circunstancias adversas en las que se ven inmersas muchas empresas actualmente, ocasionan que los trabajadores deban soportar mayores cargas de trabajo, jornadas más largas, recortes salariales y un empeoramiento de las condiciones de trabajo. Estos inconvenientes tarde o temprano pueden suponer mayores costes adicionales por absentismo, derivado de cansancio físico o enfermedades psicosomáticas. Visto así, el WE, supone una ventaja competitiva para las empresas, ya que puede ser medido y potenciado mediante intervenciones organizacionales que den como resultado trabajadores comprometidos e implicados, que realicen su trabajo con alto grado de satisfacción. Una organización «inteligente» será capaz de aplicar todas estas herramientas logrando un capital humano proactivo, innovador y con altas dosis de creatividad, contando así con trabajadores dispuestos a dar todo su potencial, lo que contribuirá a lograr los objetivos comunes. Lo ideal es que a la vez que se consiga un mayor beneficio económico para la empresa se logren los objetivos personales de los trabajadores, su desarrollo profesional y contribuir a su bienestar al realizar su trabajo.

\section{Bibliografía}

Acosta, H., Salanova, M., Llorens, S. (2011). «¿Qué prácticas organizacionales saludables son más frecuentes en las empresas? Un análisis cualitativo» disponible en http:// www.uji.es/bin/publ/edicions/jfilo/psisoc/1.pdf.

Bakker, A.B., Albrecht, S.L., Leiter, M.P. (2011): "Work engagement: Further reflections on the state of play». European Journal of Work and Organizational Psychology, 20(1), pp. 74-88. 
Chuang, C.H., LiaO, H.U.I. (2010). «Strategic human resource management in service context: Taking care of business by taking care of employees and customers». Personnel Psychology, 63(1), pp. 153-196.

Durán, M.A., Manteca, A.J. (2013). «Engagement en profesionales de la acción social. Diferencias entre Administración y Tercer Sector desde la perspectiva del modelo de demandas y recursos laborales». Documentos de Trabajo Social, 51, pp. 45-68.

Extremera, N., Durán, M.A., Rey, L. (2005). "La inteligencia emocional percibida y su influencia sobre la satisfacción vital, la felicidad subjetiva y el engagement en trabajadores de centros para personas con discapacidad intelectual». Ansiedad y estrés, 11(1), pp. 63-73.

KaHN, W.A. (1990). «Psychological conditions of personal engagement and disengagement at work». Academy of Management Journal, 33(4), pp. 692-724.

Luthans, F. (2002). "The need for and meaning of positive organizational behavior». Journal of Organizational Behavior, 23(6), pp. 695-706.

Reissner, S., Pagan, V. (2012). «Employee engagement: the use of storytelling» disponible en https://www.esrc.ac.uk/.../7c4be2d5-cb41-408b-a387-1f144f80ad3c.

Rodríguez-Muñoz, A.. Bakker, A.B. (2013). «El engagement en el trabajo». Salud laboral: riesgos laborales psicosociales y bienestar laboral, 23, pp. 437-452.

Salanova, M., Martínez, I.M., Llorens, S. (2005). «Psicología organizacional positiva». Psicologia de la Organización. Madrid: Pearson Prentice Hall, pp. 349-376.

Salanova, M., Schaufeli, W.B. (2004). «El engagement de los empleados: un reto emergente para la dirección de los recursos humanos». Estudios financieros. Revista de trabajo y seguridad social: Comentarios, casos prácticos: recursos humanos, (261), pp. 109138.

Salanova, M., Schaufeli, W. (2009) El «engagement» en el trabajo. Cuando el trabajo se convierte en pasión. Madrid: Alianza Editorial.

Schaufeli, W.B., Bakker, A.B. (2003). «Utrecht work engagement scale: Preliminary manual». Occupational Health Psychology Unit, Utrecht University, Utrecht.

Schaufeli,W. B., Salanova, M., Gonzalez-Romá, V., Bakker, A.B. (2002). "The Measurement of engagement and burnout: A confirmative analytic approach». Journal of Happiness Studies, 3, pp. 71-92.

Stirpe, L., Revilla, A.J. (2013): «Efectos de la contratación temporal sobre los resultados de los sistemas de trabajo de alto rendimiento». Universia Business Review, 39, pp. 1431.

TANG, T.W., TANG, Y. (2012). «Promoting service-oriented organizational citizenship behaviors in hotels: the role of high-performance human resource practices and organizational social climates». International Journal of Hospitality Management, 31(3), pp. 885-895.

Torrente, P., Salanova, M., Llorens, S., Schaufeli, W.B. (2012). «Teams make it work: how team work engagement mediates between social resources and performance in teams». Psicothema, 24(1), pp. 106-112. 


\section{Anexo 1. Escala Utrecht de Engagement en el Trabajo.} UWES-17 (adaptada para Aspromanis)

Encuesta de Bienestar en el Trabajo (UWES) ()

\begin{tabular}{|l|l|l|l|}
\hline \multicolumn{2}{|l|}{ CENTRO O SERVICIO } & & \\
\hline \multicolumn{2}{|l|}{ ÁMBITO PROFESIONAL } & EDAD & \\
\hline ATENCIÓN DIRECTA & & ANTIGÜEDAD & \\
\hline TÉCNICO ESPECÍFICO & & SEXO & \\
\hline ADMINISTRACIÓN & & NIVEL DE ESTUDIOS & \\
\hline SERVICIO DOMÉSTICO & & & \\
\hline
\end{tabular}

Las siguientes preguntas se refieren a los sentimientos de las personas en el trabajo. Por favor, lea cuidadosamente cada pregunta y decida si se ha sentido de esta forma. Si nunca se ha sentido así conteste «0» (cero), y en caso contrario indique cuántas veces se ha sentido así teniendo en cuenta el número que aparece en la siguiente escala de respuesta (de 1 a 6 ).

\begin{tabular}{|c|c|c|c|c|c|c|}
\hline Nunca & $\begin{array}{c}\text { Casi } \\
\text { nunca }\end{array}$ & $\begin{array}{c}\text { Algunas } \\
\text { veces }\end{array}$ & $\begin{array}{c}\text { Regular- } \\
\text { mente }\end{array}$ & $\begin{array}{c}\text { Bastantes } \\
\text { veces }\end{array}$ & $\begin{array}{c}\text { Casi } \\
\text { siempre }\end{array}$ & Siempre \\
\hline 0 & 1 & 2 & 3 & 4 & 5 & 6 \\
\hline $\begin{array}{c}\text { Ninguna } \\
\text { vez }\end{array}$ & $\begin{array}{c}\text { Pocas veces } \\
\text { al año }\end{array}$ & $\begin{array}{c}\text { Una vez } \\
\text { al mes } \\
\text { o menos }\end{array}$ & $\begin{array}{c}\text { Pocas veces } \\
\text { al mes }\end{array}$ & $\begin{array}{c}\text { Una vez } \\
\text { por semana }\end{array}$ & $\begin{array}{c}\text { Pocas veces } \\
\text { por semana }\end{array}$ & $\begin{array}{c}\text { Todos } \\
\text { los días }\end{array}$ \\
\hline
\end{tabular}




\begin{tabular}{|r|l|l|}
\hline 1 & & En mi trabajo me siento lleno de energía \\
\hline 2 & & Mi trabajo está lleno de significado y propósito \\
\hline 3 & & El tiempo vuela cuando estoy trabajando \\
\hline 4 & & Soy fuerte y vigoroso en mi trabajo \\
\hline 5 & & Estoy entusiasmado con mi trabajo \\
\hline 7 & & Cuando estoy trabajando olvido todo lo que pasa alrededor de mí \\
\hline 8 & & Mi trabajo me inspira \\
\hline 9 & & Cuando me levanto por las mańanas tengo ganas de ir a trabajar \\
\hline 10 & & Estoy orgulloso del trabajo que hago \\
\hline 11 & & Estoy inmerso en mi trabajo \\
\hline 12 & & Puedo continuar trabajando durante largos períodos de tiempo \\
\hline 13 & & Mi trabajo es retador \\
\hline 14 & & Me «dejo llevar» por mi trabajo \\
\hline 15 & & Soy muy persistente en mi trabajo \\
\hline 16 & & Me es difícil «desconectarme» de mi trabajo \\
\hline 17 & & Incluso cuando las cosas no van bien, continuo trabajando \\
\hline
\end{tabular}

